\title{
Analysis on Rehabilitation Effect of Modern Rehabilitation Concept Combined with Acupuncture and Massage on Cerebral Palsy in Children
}

\author{
Xiaosu Jie ${ }^{1}$, Haijun Shan ${ }^{1}$, Yujin Hou ${ }^{1}$, Hua Shi ${ }^{1}$, Yuanjun Lou ${ }^{1}$, Yingying Zhang ${ }^{1}$, Xin Guo ${ }^{1}$ \\ ${ }^{1}$ Henan province hospital of Traditional Chinese Medicine, Zhengzhou, Henan, 450002
}

Keywords: analysis; rehabilitation effect; modern rehabilitation concept; acupuncture and massage; cerebral palsy in children

\begin{abstract}
Objective: To observe the rehabilitation effect of modern rehabilitation concept combined with TCM intervention on cerebral palsy in children. Methods: A total of 89 children with cerebral palsy in our hospital were selected as study subjects and randomly divided into observation group $(n=45)$ and control group $(n=44)$. The control group used modern rehabilitation concepts to intervene. The observation group adopted modern rehabilitation concepts combined with comprehensive Chinese medicine interventions to compare the two groups before and after treatment of limb motor function (GMFM score evaluation), daily life ability (Barthel index evaluation) and total treatment efficiency. Results: The difference of GMFM score and BI index between the two groups before intervention was not statistically significant $(\mathrm{P}>0.05)$. After the intervention, the above two indicators were both increased, and there was a significant difference between the two groups $(\mathrm{P}<0.05)$. The scores of GMFM and $\mathrm{BI}$ in the observation group were higher than those in the control group after intervention $(\mathrm{P}<0.05)$, suggesting that the improvement of limb motor function and daily living ability was greater. Conclusion: The concept of modern rehabilitation combined with the comprehensive intervention of TCM in children with cerebral palsy has a satisfactory rehabilitation effect, which is conducive to the recovery of limb movement function and daily living ability. It is easy to operate and has low treatment cost, and it is worthy of promotion and application.
\end{abstract}

\section{Introduction}

Pediatric cerebral palsy, also known as pediatric brain-derived spasm, refers to an abnormal brain development during maternal development or within one month after birth, leading to central nervous system disorders, postural developmental disorders, and limited mobility. Cerebral palsy will not only affect the children's actions and intelligence, but also cause visual, auditory, language barriers and epilepsy. According to the latest screening data of the China-America International Institute of Cerebral Palsy in the first quarter of 2014, there are nearly 6 million children with cerebral palsy in China. Each year, 30,000 to 40,000 children with cerebral palsy are newly diagnosed. Because of the lack of awareness about cerebral palsy in society, the morbidity of children with cerebral palsy in China reaches $42 \%$ to $45 \%$ [1]. Therefore, it is of great significance to study the clinical efficacy of treating cerebral palsy in children.

\section{The Concept of Modern Rehabilitation Combined with Acupuncture and Massage for Rehabilitation of Cerebral Palsy in Children}

The comprehensive therapy based on scalp acupuncture He Weijie and other three-needle acupuncture combined with home education 150 cases of $\mathrm{CP}$, the main point to take four needles, brain three needles, three needles, mentally retarded persons with intellectual three-pin, flowing murmurs and unclear speeches, three needles, three limbs, three limbs, three needles, etc., needle retention 30 minutes, once a day, the whole course of 4 months, and according to clinical dialectical points were given acupuncture injection, results markedly effective in 26 cases, effective in 101 cases, Ineffective in 23 cases, Total effective rate was 84.6\%. Yuan Haibin[2] Observed 118 cases of $\mathrm{CP}$ by using three needles plus exercise therapy. The scalp acupuncture used three needles, three 
needles, four needles, and three brains. The body needle was added with three needles. 3 to $4 \mathrm{~h}$ of needle insertion, half-way stimulation (except epilepsy history) stimulation for 20 min, results markedly effective in 51 cases, effective in 57 cases, invalid in 10 cases, total effective rate of $91.7 \%$, significantly better than the exercise group (exercise $73.1 \%$ of the therapy group). Kang Yuxin [3] used scalp acupuncture to treat 46 cases of CP and had satisfactory results in recovery of motor function. The main points were three needles, five needles, and three pre-exercise needles. Puncturing or retention was adopted according to the age, and the needle retention period was rapid. The needles were performed 3 times, 14 cases were markedly effective, 23 cases were effective, 9 cases were ineffective, and the total effective rate was $80.4 \%$. Peng Guilan et al. used scalp acupuncture combined with acupoint injection to observe the incidence of cerebral palsy in children. The head needle took the first three needles of the fontanelle, Sishencong, and retained the needle for 20 minutes. The acupuncture point injection used Baihui and Sishencong, and the upper limbs selected the shoulder and Quchi. Hegu, taking Zusanli, Venezuela, and Chengfu from the lower extremities, medicinal vitamins B1, B12, citicoline, and intravenous infusion of cerebrolysin and compound salvia miltiorrhiza injection, the results showed that the early treatment of the acupuncture points, the incidence of cerebral palsy Lower than the control group (only intravenous infusion of cerebrolysin and compound Danshen injection). Liu Zhenluo and others took the scalp acupuncture as the main treatment for 210 cases of CP, from the Shen Ting point along the piercing of Baihui to Baihui, from Baihui to the head of the brain, and the top acupuncture to Xuan $\mathrm{Li}$ (bilateral) as the basic constituency, if the upper limb paralysis. Choose from Baihui to puncture and collateral, from the Baihui to the front side of the acupoint line outside the 2.25 inches (two sides); and according to different symptoms use different areas, once every other day, every time the needle 4h, choose to sparse Mibo alternately treated with electro-acupuncture for 15 minutes, combined with medical treatment and physical training. The treatment results showed that 82 cases were markedly effective, 67 cases were effective, 61 cases were ineffective, and the total effective rate was $71 \%$. Li Huilan and others used scalp acupuncture combined with modern rehabilitation therapy CP, the main point to take Sishen Cong, Baihui, sports area, feeling area, etc., Xu dynamic type plus sense of movement and balance area, language barrier plus language area, saliva flow Add to the warehouse, buccal car. Acupuncture did not use strong stimulation, retained the needle for $1 \mathrm{~h}$, and simultaneously performed rehabilitation training once a day. After treatment, the ADL score of the treatment group was significantly better than that of the control group (using modern rehabilitation therapy). Zhang Haiting used scalp acupuncture as the main method to treat 100 cases of CP. The exercise area, sensory area, and language area were selected. After 30 minutes of needle retention, the needle was quickly rotated for 30 seconds, and the needle was continued for 30 minutes. Through external barriers, Quchi, Sanyinjiao, etc., together with acupoint closure, physiotherapy and other therapies, the efficacy evaluation showed that: 28 cases were markedly effective, 68 cases were effective, 4 cases were ineffective, and the total effective rate was 96\%, which was significantly better than that of the control group (physiotherapy, Drugs, massage methods combined) [4].

\section{Body Acupuncture-Based Comprehensive Therapy}

Zhao Yaodong treated 30 cases of CP with Wen Tong acupuncture, the main points were taken from Fengchi, Baihui, Sishencong, Shenshu, etc., and the points were dialectically differentiated. After the needle was inserted, the right thumb was continuously forwarded 9 times, and the needle was sinking. Immediately after the tip of the needle, pull the sensing site for continuous reinsertion and gently raise the needle 9 times to continue to tighten the needle. Operate for 60 seconds per hole and retain the needle for 30 minutes. The curative effect shows that 20 cases are markedly effective, 6 cases are effective, 2 cases are improved, and 2 cases are invalid. The total effective rate is $93.33 \%$. He Jun took 30 cases of Xingnao Kaiqiao acupuncture treatment, and took Neiguan, Renzhong, Sishencong, Quchi, Hegu, Sanyinjiao, etc. in the morning, and took the wind pool, finished bone, Tianzhu, and Huatuo clip spines in the afternoon. The needle was retained for $20 \mathrm{~min}$ for 3 consecutive months. The results showed that 2 cases were cured, 12 cases were markedly 
effective, 14 cases improved and 2 cases were ineffective. The total effective rate was 93.3\%. Zhang Xiaoli et al. observed 354 cases of CP with acupuncture at Jiaji ridge. The acupoints were Jiaji, 17 vertebrae, and Changqiang acupoints. They were oblique or flat. The direction was toward the brain. The needle was retained for $30 \mathrm{~min}$. On one day, if there was no twitch stimulation with electroacupuncture, 84 cases were markedly effective after treatment, 241 cases were effective, 29 cases were ineffective, and the total effective rate was $91.8 \%$. Li Hongchao used acupuncture Tongdu method to treat 325 cases of CP. The acupoints were mainly formed by the Governor Vessel. The hand, foot, and the solar system were supplemented by Shaoyang Jing and Yangming Jing. The main points of the head were Sishencong and Baihui. The body acupuncture was taken at Hegu, Waiguan, Boneless, Taichong and so on. The needle was retained for $45 \mathrm{~min}$, and the acupoints were discriminated. The results of the treatment were clinically cured in 261 cases, improved in 27 cases, and invalid in 37 cases. The total effective rate was $88.6 \%$.

\section{Other Comprehensive Therapy}

Lou Weihong treated 214 cases of CP with ear acupuncture treatment. He used sympathetic, thalamus, subcortical, and other primary points of the auricular acupuncture to administer acupuncture, knee, and elbow. At the same time, massage techniques were used in conjunction with functional training. The results showed that: Among the 101 patients whose motor function was close to or normal, there were 97 patients with abnormal motor function, but still abnormal muscle tension, and abnormal posture. There were 16 cases without improvement, and the total effective rate was 92.5\%. Guan Zunhui used tongue and needle as the main treatment for CP112 cases, tongue needle coring, spleen, liver and other points, Jinjin, jade fluid, etc., applied reinforcing diarrhea without retention needles, scalp acupuncture to take advantage of brain 16 points, retention needle 20 minutes, and with the card points, after treatment basically recovered 21 cases, markedly improved in 44 cases, improved in 40 cases, ineffective in 7 cases, the total effective rate was 93.75\%. Electroacupuncture was based on scalp acupuncture and body acupuncture was used as a supplement. The head was taken from exercise area, sensory area, verbal area, etc. The main body acupuncture was taken by foot Yangming meridians. Foot and lower yin through the foot, the foot through the sun; water needle acupuncture points, medicinal brain protein hydrolysis injection alternate acupoints, cured in 2 cases, improved in 123 cases, 7 cases ineffective, the total effective rate was $94.7 \%$. He Guangyuan and other massage head and ridge push treatment $\mathrm{CP}$, the head to get the five classics, scalp sports area, feeling area, etc., and finally click Baihui, Sishencong, Fengfu and other points, the technique should be gentle; spine along the Governor The veins and the bladders on both sides are clicked from top to bottom, and a fingernail is used to massage the full moon. The technique is stronger. Finally, the vibrator method is applied to the spine from top to bottom, and the massage is performed along with the acupoints, along with functional training and medical treatment. Twenty-three cases were treated, 12 cases were markedly effective, 10 cases were effective, and 1 case was ineffective. The total effective rate was 95.7 percent [5].

\section{Analysis of Rehabilitation Effect of Modern Rehabilitation Concept Combined with Acupuncture and Massage on Cerebral Palsy in Children}

Pediatric cerebral palsy is a category of "five late" and "five soft" categories in traditional Chinese medicine. Chinese medicine believes that this disease is mainly caused by lack of congenital fetal degeneration, lack of parental qi and blood, conceitedness, and incompetence of the parents, resulting in inadequate filling of the brain, insufficient blood and blood, and retarded growth. Under the guidance of the overall concept of traditional Chinese medicine and syndrome differentiation and treatment, traditional Chinese medicine therapy has a good effect in the rehabilitation process of cerebral palsy, especially acupuncture and moxibustion therapy. It is one of the ideal methods in the current treatment. However, the traditional Chinese medicine has not formulated a unified standard for the diagnosis of cerebral palsy and evaluation standards for therapeutic efficacy, which hinders the further study of cerebral palsy. Therefore, traditional 
Chinese medicine should be combined with modern rehabilitation theory to draw on the advantages of modern rehabilitation medicine, especially rehabilitation assessment and its unique treatment methods such as PT, OT, and ST, so that Chinese and Western rehabilitation therapy can be organically combined to improve the diagnosis and treatment of cerebral palsy. Let millions of children with cerebral palsy grow healthily.

Causes of cerebral palsy in children include maternal (premature mother, recurrent miscarriage, mental illness), postnatal factors (convulsions, persistent jaundice, exchange transfusion therapy), acquired factors (high fever, brain trauma, meningitis, intracranial hemorrhage). In three aspects, pediatric cerebral hypoplasia, hypoxia, bleeding, water, infarction, softening and atrophy eventually lead to cerebral palsy; jaundice abnormalities mainly affect the pediatric central nervous system, improper treatment or not timely may also induce cerebral palsy. This study was to explore the effect of TCM intervention in the rehabilitation of children with cerebral palsy, and to compare the effects of simple modern rehabilitation measures with that of combined TCM interventions. The results showed that both the GMFM score and the BI index of the combined group were significantly increased. Limb motor function and daily living ability were greatly improved, and the total effective rate of treatment was $88.9 \%$ (40/45), which was higher than that of the control group (70.5\%) (31/44). The difference was statistically significant $(\mathrm{P}<0$. 05) Obvious advantages. According to modern medical theory, pediatric cerebral palsy is a motor dysfunction caused by brain lesions. The rehabilitation method is based on the theory of central nervous system plasticity, including physiotherapy, exercise therapy, occupational therapy, etc., with inhibitory techniques, slamming techniques, and key Regional control and other reflexes to suppress abnormal posture and exercise patterns for rehabilitation training to induce the body to produce reflex movements, and the body induced the band to cause oppressive stimulation, abnormal reflex pathways are suppressed, and the normal motor development of children is promoted.

Chinese medicine believes that children with cerebral palsy belong to the categories of "fetal weakness”, “five hard”, “five soft”, “five late”, congenital fetal deafness, deficiency of kidney yang, or muscles of muscles and muscles due to heat poisoning, lack of sperm and blood, the lack of a brain stem cannot be caused by warm organs and raised bones. Judging from the meridian point of view, it is mostly thought to be caused by the damage of the Governor Vessel or the abnormality of the pulsed gas. Acupuncture and moxibustion is a unique treatment for traditional Chinese medicine. It can clear the meridians, reconcile yin and yang, communicate inside and outside, replenishing liver and kidney, and invigorating yang to improve the human microcirculation. Scalp acupuncture, such as the supine position of the anterior oblique line, the frontal midline, etc., the prone position of the supraorbital posterior oblique line, Sishencong, etc. can form benign stimuli, increase cerebral cortical blood supply, improve brain tissue oxygen carrying capacity, and damage The neurons are thus repaired and regenerated, and the function of the damaged area of the brain is compensated. Body acupoint selection is based on the Yang Ming Jing, supplemented by the sun, Shao Yang and other classics and each point echoes each other, prompting the body to meridians, blood circulation. Massage through the points to achieve the adjustment of organs, qi and blood, dredge the meridians, balance of yin and yang, and ultimately promote the children's physical fitness, body immunity enhancement, is a health method, with the help of massage to promote microvascular, cerebrovascular and lymphatic circulation, accelerate metabolism, and finally Blood circulation, Sanjie pain. Modern medical studies have shown that massage can cause direct stimulation of muscles and nerve tissue, nutrition of nerves and muscles, promote its elastic recovery and reduce muscle tension, help muscle balance, eliminate joint contracture, bone and joint stability and activity as a result, the children in the observation group had better physical activity and daily living ability.

\section{Conclusion}

In summary, the concept of modern rehabilitation combined with the comprehensive intervention of TCM in children with cerebral palsy has a satisfactory rehabilitation effect, which facilitates the recovery of limb motor function and daily living ability. It is easy to operate and has low treatment 
cost. It is worthy of promotion and application.

\section{References}

[1] He Yujie, Xia Guohua, Yin Yuxi. "Zhu three needles" combined with home education for the treatment of pediatric cerebral palsy [J]. Guangdong Medical Science, 2008, 19 (11): 881

[2] Yuan Haibin. Observation of Curative Effect of 118 Cases of Cerebral Palsy in Children Treated by "Zhi San" [J]. Modern Rehabilitation, 2001, 9, 5(9):84

[3] Kang Yuxin. Observation of the therapeutic effect of scalp acupuncture on children with cerebral palsy[J]. Chinese Medicine,2005,7,3(7):53

[4] Peng Guilan, Zhang Ling. Observe the incidence of cerebral palsy in children with scalp acupuncture combined with point injection [J]. Chinese Acupuncture, 2003, 5, 23(5): 263

[5] Liu Zhendi, Zhang Hongyan. Clinical observation of 210 cases of cerebral palsy in children treated with scalp acupuncture [J]. Chinese Acupuncture, 2009, 11:651 